\title{
Atomic data from the Iron Project
}

\section{Photoionization cross sections and oscillator strengths for Ni II}

\author{
M.A. Bautista \\ Laboratory for High Energy Astrophysics, Code 662, NASA Goddard Space Flight Center, Greenbelt, MD 20771, U.S.A. \\ Internet: bautista@lhea1.gsfc.nasa.gov
}

Received April 6; accepted April 8, 1999

\begin{abstract}
We present calculations of energy levels, photoionization cross sections and dipole allowed transition probabilities for singly ionized nickel. The computations were carried out in the close coupling approximation using the R-matrix method including $49 \mathrm{LS}$ terms with multiplicity $(2 S+1)=1,3$, and 5 of the target ion Ni III. We calculated $836 \mathrm{LS}$ states, 561 of which are bound, with principal quantum number $n \leq 10$ and angular momentum $L \leq 7$. The results also include dipole oscillator strengths for 23738 LS transitions, and photoionization cross sections with detailed resonance structures for all bound states. The $f$-values and radiative lifetimes agree well with available experimental measurements. However, the present $f$-values disagree considerably from previous computations by Kurucz and coworkers which are currently used in Ni II abundance determination of the ISM. In particular, the Kurucz $f$-values for transitions from the $\mathrm{Ni}$ II ground state seem to be overestimated by factors of two to three thus, nickel may be less depleted in ISM than previously estimated. The photoionization cross section for the $3 \mathrm{~d}^{9}{ }^{2} \mathrm{D}$ ground state of Ni II exhibits near threshold packs of resonances that rise over the background cross section by several orders of magnitude.
\end{abstract}

Key words: atomic data - atomic processes - line: formation - ISM: abundances — ultraviolet: general — infrared: ISM: lines and bands

\section{Introduction}

Accurate atomic data for iron and other iron group elements is of major importance in astrophysics. Among these elements, nickel is the second most abundant element after iron. Then, reliable modeling of the spectra of this element and determination of its abundance have direct implications on our understanding of the chemical evolution of the universe. In particular, singly ionized nickel is often detected in studies of absorption lines in the diffuse ISM. Ni II is also a common source of emission lines in the spectra of H II regions, novae, supernovae, and supernova remnants. Despite of the importance of nickel in astrophysics very few studies of the atomic data for this element have ever been carried out. Consequently, there are at present great uncertainties on the gas phase abundances of nickel which reach up to orders of magnitude (e.g. Haas et al. 1996; Oliva et al. 1988; Henry \& Fesen 1988).

The IRON Project is an international collaboration devoted to the computation of accurate atomic data for iron peak elements (Hummer et al. 1993). A complete list of papers including those in press can be found at http://www.am.qub.uk/projects/iron/papers/, where abstracts are also given for each paper. In the present paper we report extensive radiative data for Ni II. The data sets include multiplet energy levels, dipole allowed transition probabilities, and total and partial photoionization cross sections including detailed resonance structures.

\section{Target expansion}

In the close coupling (CC) approximation the total wave function of the electron-ion system is represented as

$\Psi(E ; S L \pi)=A \sum_{i} \chi_{i} \theta_{i}+\sum_{j} c_{j} \Phi_{j}$,

where $\chi_{i}$ is the target ion wave function in a specific state $S_{i} L_{i}, \theta_{i}$ is the wave function for the free electron, and $\Phi_{j}$ are short range correlation functions for the bound $(\mathrm{e}+\mathrm{ion})$ system. Accurate CC calculations of atomic processes require, first of all, a good representation of the target ion. For complex ions an accurate representation must include a large number of correlation configurations. However, in order for the computations to be computationally tractable, the configuration expansion must be carefully and economically chosen. 
Table 1. Calculated and observed term energies (Rydbergs) for Ni III relative to the $3 \mathrm{~d}^{8}\left({ }^{3} \mathrm{~F}\right)$ ground state. The spectroscopic and correlation configurations for Ni III, and the values of the scaling parameters $\lambda_{n l}$ for each orbital in the Thomas-Fermi-Dirac potential used in Superstructure, are also given

\begin{tabular}{|c|c|c|c|c|c|c|c|c|c|}
\hline & Level & & $\mathrm{E}_{\mathrm{cal}}$ & $\mathrm{E}_{\text {obs }}$ & & Level & & $\mathrm{E}_{\mathrm{cal}}$ & $\mathrm{E}_{\text {obs }}$ \\
\hline 1 & $3 d^{8}$ & ${ }^{3} \mathrm{~F}$ & 0.000000 & 0.000000 & 2 & $3 d^{8}$ & ${ }^{1} \mathrm{D}$ & 0.151090 & 0.118808 \\
\hline 3 & $3 d^{8}$ & ${ }^{3} \mathrm{P}$ & 0.169259 & 0.144311 & 4 & $3 d^{8}$ & ${ }^{1} \mathrm{G}$ & 0.230022 & 0.201524 \\
\hline 5 & $3 d^{8}$ & ${ }^{1} \mathrm{~S}$ & 0.562995 & 0.469649 & 6 & $3 \mathrm{~d}^{7}\left({ }^{4} \mathrm{~F}\right) 4 \mathrm{~s}$ & ${ }^{5} \mathrm{~F}$ & 0.436503 & 0.490628 \\
\hline 7 & $3 \mathrm{~d}^{7}\left({ }^{4} \mathrm{~F}\right) 4 \mathrm{~s}$ & ${ }^{3} \mathrm{~F}$ & 0.510200 & 0.558378 & 8 & $3 \mathrm{~d}^{7}\left({ }^{4} \mathrm{P}\right) 4 \mathrm{~s}$ & ${ }^{5} \mathrm{P}$ & 0.620387 & 0.640931 \\
\hline 9 & $3 d^{7}\left({ }^{2} G\right) 4 s$ & ${ }^{3} \mathrm{G}$ & 0.644262 & 0.679739 & 10 & $3 d^{7}\left({ }^{4} \mathrm{P}\right) 4 \mathrm{~s}$ & ${ }^{3} \mathrm{P}$ & 0.696886 & 0.705399 \\
\hline 11 & $3 d^{7}\left({ }^{2} G\right) 4 s$ & ${ }^{1} \mathrm{G}$ & 0.681025 & 0.713123 & 12 & $3 \mathrm{~d}^{7}\left({ }^{2} \mathrm{P}\right) 4 \mathrm{~s}$ & ${ }^{3} \mathrm{P}$ & 0.706707 & 0.715485 \\
\hline 13 & $3 d^{7}\left({ }^{2} \mathrm{H}\right) 4 \mathrm{~s}$ & ${ }^{3} \mathrm{H}$ & 0.706085 & 0.739700 & 14 & $3 d^{7}\left(a^{2} D\right) 4 s$ & ${ }^{3} \mathrm{D}$ & 0.727877 & 0.742560 \\
\hline 15 & $3 d^{7}\left({ }^{2} \mathrm{P}\right) 4 \mathrm{~s}$ & ${ }^{1} \mathrm{P}$ & 0.743514 & 0.761912 & 16 & $3 \mathrm{~d}^{7}\left({ }^{2} \mathrm{H}\right) 4 \mathrm{~s}$ & ${ }^{1} \mathrm{H}$ & 0.742028 & 0.773121 \\
\hline 17 & $3 d^{7}\left(a^{2} D\right) 4 s$ & ${ }^{1} \mathrm{D}$ & 0.765820 & 0.780518 & 18 & $3 d^{7}\left({ }^{2} F\right) 4 s$ & ${ }^{3} \mathrm{~F}$ & 0.890389 & 0.884557 \\
\hline 19 & $3 d^{7}\left({ }^{2} F\right) 4 s$ & ${ }^{1} \mathrm{~F}$ & 0.929649 & 0.920024 & 20 & $3 d^{7}\left({ }^{4} F\right) 4 p$ & ${ }^{5} \mathrm{~F}^{\mathrm{O}}$ & 0.937097 & 1.00141 \\
\hline 21 & $3 d^{7}\left({ }^{4} F\right) 4 p$ & ${ }^{5} \mathrm{D}^{\circ}$ & 0.951207 & 1.01975 & 22 & $3 d^{7}\left({ }^{4} F\right) 4 p$ & ${ }^{5} \mathrm{G}^{\mathrm{o}}$ & 0.957573 & 1.02468 \\
\hline 23 & $3 d^{7}\left({ }^{4} F\right) 4 p$ & ${ }^{3} \mathrm{G}^{\circ}$ & 0.985697 & 1.05115 & 24 & $3 d^{7}\left({ }^{4} F\right) 4 p$ & ${ }^{3} \mathrm{~F}^{\mathrm{o}}$ & 0.995254 & 1.05715 \\
\hline 25 & $3 d^{7}\left({ }^{4} F\right) 4 p$ & ${ }^{3} \mathrm{D}^{\circ}$ & 1.018847 & 1.07862 & 26 & $3 d^{7}\left({ }^{4} \mathrm{P}\right) 4 \mathrm{p}$ & ${ }^{5} \mathrm{~S}^{\circ}$ & 1.072974 & 1.10526 \\
\hline 27 & $3 d^{7}\left({ }^{4} \mathrm{P}\right) 4 \mathrm{p}$ & ${ }^{5} \mathrm{D}^{\circ}$ & 1.142424 & 1.17636 & 28 & $3 d^{7}\left({ }^{4} \mathrm{P}\right) 4 \mathrm{p}$ & ${ }^{3} \mathrm{~S}^{\circ}$ & 1.164764 & 1.18346 \\
\hline 29 & $3 d^{7}\left({ }^{2} G\right) 4 p$ & ${ }^{3} \mathrm{H}^{\circ}$ & 1.145107 & 1.19329 & 30 & $3 d^{7}\left({ }^{2} G\right) 4 p$ & ${ }^{3} \mathrm{~F}^{\circ}$ & 1.154800 & 1.20136 \\
\hline 31 & $3 d^{7}\left({ }^{4} P\right) 4 p$ & ${ }^{5} \mathrm{P}^{\mathrm{o}}$ & 1.170775 & 1.20340 & 32 & $3 d^{7}\left({ }^{2} \mathrm{P}\right) 4 \mathrm{p}$ & ${ }^{3} \mathrm{P}^{\mathrm{o}}$ & 1.184242 & 1.20688 \\
\hline 33 & $3 d^{7}\left({ }^{2} G\right) 4 p$ & ${ }^{1} \mathrm{G}^{\mathrm{O}}$ & 1.156253 & 1.20589 & 34 & $3 d^{7}\left({ }^{4} \mathrm{P}\right) 4 \mathrm{p}$ & ${ }^{3} \mathrm{D}^{\circ}$ & 1.176099 & 1.20764 \\
\hline 35 & $3 d^{7}\left({ }^{2} G\right) 4 p$ & ${ }^{3} \mathrm{G}^{\circ}$ & 1.162174 & 1.21295 & 36 & $3 d^{7}\left({ }^{2} G\right) 4 p$ & ${ }^{1} \mathrm{H}^{\mathrm{o}}$ & 1.161474 & 1.21402 \\
\hline 37 & $3 d^{7}\left({ }^{2} G\right) 4 p$ & ${ }^{1} \mathrm{~F}^{\mathrm{o}}$ & 1.178121 & 1.22136 & 38 & $3 d^{7}\left({ }^{4} P\right) 4 p$ & ${ }^{3} \mathrm{P}^{\mathrm{o}}$ & 1.199152 & 1.22660 \\
\hline 39 & $3 d^{7}\left({ }^{2} P\right) 4 p$ & ${ }^{3} \mathrm{D}^{\circ}$ & 1.219001 & 1.23933 & 40 & $3 d^{7}\left({ }^{2} \mathrm{H}\right) 4 \mathrm{p}$ & ${ }^{3} \mathrm{G}^{\circ}$ & 1.200922 & 1.24696 \\
\hline 41 & $3 \mathrm{~d}^{7}\left({ }^{2} \mathrm{H}\right) 4 \mathrm{p}$ & ${ }^{3} \mathrm{I}^{\mathrm{o}}$ & 1.211617 & 1.24677 & 42 & $3 d^{7}\left({ }^{2} G\right) 4 p$ & ${ }^{1} \mathrm{~S}^{\circ}$ & 1.223161 & 1.24983 \\
\hline 43 & $3 d^{7}\left({ }^{2} G\right) 4 p$ & ${ }^{3} \mathrm{D}^{\circ}$ & 1.227314 & 1.25616 & 44 & $3 d^{7}\left({ }^{4} \mathrm{P}\right) 4 \mathrm{p}$ & ${ }^{1} \mathrm{I}^{\mathrm{o}}$ & 1.211617 & 1.26338 \\
\hline 45 & $3 d^{7}\left({ }^{2} \mathrm{P}\right) 4 \mathrm{p}$ & ${ }^{3} \mathrm{~F}^{\circ}$ & 1.239091 & 1.27101 & 46 & $3 d^{7}\left({ }^{2} \mathrm{H}\right) 4 \mathrm{p}$ & ${ }^{3} \mathrm{~S}^{\circ}$ & 1.257660 & 1.27478 \\
\hline 47 & $3 d^{7}\left({ }^{2} \mathrm{H}\right) 4 \mathrm{p}$ & ${ }^{1} \mathrm{P}^{\mathrm{o}}$ & 1.253598 & 1.27960 & 48 & $3 d^{7}\left({ }^{2} G\right) 4 p$ & ${ }^{3} \mathrm{H}^{\circ}$ & 1.242199 & 1.28986 \\
\hline 49 & $3 d^{7}\left({ }^{2} G\right) 4 p$ & ${ }^{1} \mathrm{D}^{\circ}$ & 1.267330 & 1.28890 & & & & & \\
\hline
\end{tabular}

Ni III configurations.

Spectroscopic: $3 \mathrm{p}^{6} 3 \mathrm{~d}^{8}, 3 \mathrm{p}^{6} 3 \mathrm{~d}^{7} 4 \mathrm{~s}, 3 \mathrm{p}^{6} 3 \mathrm{~d}^{7} 4 \mathrm{p}$.

Correlation: $3 p^{6} 3 d^{6} 4 s^{2}, 3 p^{6} 3 d^{6} 4 p^{2}, 3 p^{4} 3 d^{8} 4 s^{2}, 3 p^{5} 3 d^{7} 4 s^{2}, 3 p^{6} 3 d^{7} 4 d, 3 p^{5} 3 d^{8} 4 d^{1}, 3 p^{6} 3 d^{6} 4 s 4 p^{1}, 3 p^{4} 3 d^{8} 4 p^{2}, 3 p^{5} 3 d^{8} 4 p^{1}$, $3 \mathrm{p}^{5} 3 \mathrm{~d}^{7} 4 \mathrm{~s}$.

$\lambda_{n l}$ : 1.43121(1s), 0.93950(2s), 1.28641(2p), 1.15714(3s), 1.02417(3p), 1.01626(3d), 0.97488(4s), 0.96711(4p), 1.26086(4d).

The CC expansion for this calculation includes 49 LS terms of the target ion Ni III. The atomic structure code SUPERSTRUCTURE (Eissner et al. 1974; Eissner 1991) was used to compute eigenfunctions for the target states dominated by the configurations $3 \mathrm{~d}^{8}, 3 \mathrm{~d}^{7} 4 \mathrm{~s}$, and $3 \mathrm{~d}^{7} 4 \mathrm{p}$. Table 1 presents the complete list of states included in the target, as well as a comparison between the calculated target term energies and the observed energies, averaged over the fine structure, taken from Sugar \& Corliss (1985). The agreement between the energies is good; in all but the lowest five terms the agreement with the experimental values is better than $9 \%$. The overall agreement is approximately $5 \%$. Another indicator of the accuracy of the target representation is the good agreement between the length and the velocity oscillator strengths ( $f$-values), which for the present case is typically $10-20 \%$.

\section{Radiative calculations}

The present calculations were carried out in LS coupling, since it is expected relativistic effects in photoionizing the low ionization stages of iron peak elements should be small. Although they might be significant for some transitions, it would be impractical at this stage to carry through a large-scale relativistic calculation involving a number of channels several times larger than the already huge value used in the LS coupling case. Also, the inclusion of fine structure has only a marginal effect on the calculation of the Rosseland or the Planck mean opacities (Seaton et al. 1994).

The second summation in the CC expansion (Eq. (1) represents short range correlation functions. These functions are very important in obtaining accurate $(\mathrm{e}+$ ion $)$ 
Table 2. Comparison between calculated energies for Ni II, $E_{\text {cal }}$, and observed energies, $E_{\text {obs }}$, from Sugar \& Corliss (1985)

\begin{tabular}{|c|c|c|c|c|c|c|c|}
\hline Conf. & Term & $E_{\text {cal }}$ & $E_{\text {obs }}$ & Conf. & Term & $E_{\text {cal }}$ & $E_{\text {obs }}$ \\
\hline $3 \mathrm{~d}^{9}$ & ${ }^{2} \mathrm{D}$ & -1.33361 & -1.28754 & $3 d^{7} 4 s^{2}$ & ${ }^{2} \mathrm{~F}$ & -0.494980 & -0.415613 \\
\hline $3 d^{8}\left({ }^{3} F\right) 4 s$ & ${ }^{4} \mathrm{~F}$ & -1.25389 & -1.25463 & $3 \mathrm{~d}^{8}\left({ }^{3} \mathrm{~F}\right) 5 \mathrm{~s}$ & ${ }^{2} \mathrm{~F}$ & -0.482026 & -0.483061 \\
\hline $3 d^{8}\left({ }^{3} F\right) 4 s$ & ${ }^{2} \mathrm{~F}$ & -1.20990 & -1.20912 & $3 \mathrm{~d}^{7}\left({ }^{4} \mathrm{~F}\right) 4 \mathrm{~s} 4 \mathrm{p}\left({ }^{3} \mathrm{P}^{\mathrm{o}}\right)$ & ${ }^{4} \mathrm{~F}^{\mathrm{O}}$ & -0.474666 & -0.512558 \\
\hline $3 d^{8}\left({ }^{3} P\right) 4 s$ & ${ }^{4} \mathrm{P}$ & -1.12102 & -1.08688 & $3 \mathrm{~d}^{7}\left({ }^{4} \mathrm{~F}\right) 4 \mathrm{~s} 4 \mathrm{p}\left({ }^{3} \mathrm{P}^{\mathrm{o}}\right)$ & ${ }^{4} \mathrm{G}^{\mathrm{o}}$ & -0.469695 & -0.511031 \\
\hline $3 d^{8}\left({ }^{1} \mathrm{D}\right) 4 \mathrm{~s}$ & ${ }^{2} \mathrm{D}$ & -1.11520 & -1.09062 & $3 \mathrm{~d}^{7}\left({ }^{4} \mathrm{~F}\right) 4 \mathrm{~s} 4 \mathrm{p}\left({ }^{3} \mathrm{P}^{\mathrm{o}}\right)$ & ${ }^{4} \mathrm{D}^{\mathrm{o}}$ & -0.453751 & -0.492245 \\
\hline $3 d^{8}\left({ }^{3} P\right) 4 s$ & ${ }^{2} \mathrm{P}$ & -1.07261 & -1.04413 & $3 \mathrm{~d}^{7}\left({ }^{4} \mathrm{~F}\right) 4 \mathrm{~s} 4 \mathrm{p}\left({ }^{3} \mathrm{P}^{\mathrm{o}}\right)$ & ${ }^{2} \mathrm{G}^{\mathrm{o}}$ & -0.437085 & -0.460033 \\
\hline $3 d^{8}\left({ }^{1} G\right) 4 s$ & ${ }^{2} \mathrm{G}$ & -1.04270 & -1.00079 & $3 \mathrm{~d}^{8}\left({ }^{3} \mathrm{~F}\right) 4 \mathrm{~d}$ & ${ }^{4} \mathrm{D}$ & -0.434582 & -0.435192 \\
\hline $3 d^{7} 4 s^{2}$ & ${ }^{4} \mathrm{~F}$ & -0.863763 & -0.867766 & $3 d^{8}\left({ }^{3} F\right) 4 d$ & ${ }^{4} \mathrm{P}$ & -0.431959 & -0.432250 \\
\hline $3 d^{8}\left({ }^{3} F\right) 4 p$ & ${ }^{4} \mathrm{D}^{\mathrm{o}}$ & -0.860164 & -0.865801 & $3 \mathrm{~d}^{8}\left({ }^{3} \mathrm{~F}\right) 4 \mathrm{~d}$ & ${ }^{4} \mathrm{H}$ & -0.422927 & -0.431218 \\
\hline $3 d^{8}\left({ }^{3} F\right) 4 p$ & ${ }^{4} \mathrm{G}^{\mathrm{o}}$ & -0.846567 & -0.853207 & $3 d^{8}\left({ }^{3} F\right) 4 d$ & ${ }^{2} \mathrm{H}$ & -0.418282 & -0.421943 \\
\hline $3 d^{8}\left({ }^{3} F\right) 4 p$ & ${ }^{4} \mathrm{~F}^{\mathrm{o}}$ & -0.834309 & -0.841718 & $3 d^{8}\left({ }^{3} F\right) 4 d$ & ${ }^{2} \mathrm{P}$ & -0.430395 & -0.427149 \\
\hline $3 d^{8}\left({ }^{3} F\right) 4 p$ & ${ }^{2} \mathrm{G}^{\mathrm{O}}$ & -0.830713 & -0.830383 & $3 d^{8}\left({ }^{3} F\right) 4 d$ & ${ }^{4} \mathrm{G}$ & -0.419595 & -0.428366 \\
\hline $3 d^{8}\left({ }^{3} F\right) 4 p$ & ${ }^{2} \mathrm{~F}^{\mathrm{o}}$ & -0.813347 & -0.821954 & $3 d^{8}\left({ }^{3} F\right) 4 d$ & ${ }^{4} \mathrm{~F}$ & -0.427318 & -0.427868 \\
\hline $3 d^{8}\left({ }^{3} F\right) 4 p$ & ${ }^{2} \mathrm{D}^{\mathrm{o}}$ & -0.811142 & -0.809619 & $3 d^{8}\left({ }^{3} F\right) 4 d$ & ${ }^{2} \mathrm{~F}$ & -0.426341 & -0.426020 \\
\hline $3 d^{8}\left({ }^{3} P\right) 4 p$ & ${ }^{4} \mathrm{P}^{\mathrm{o}}$ & -0.731683 & -0.707929 & $3 d^{8}\left({ }^{3} F\right) 4 d$ & ${ }^{2} \mathrm{G}$ & -0.423523 & -0.417034 \\
\hline $3 d^{8}\left({ }^{1} D\right) 4 p$ & ${ }^{2} \mathrm{~F}^{\mathrm{o}}$ & -0.719775 & -0.695865 & $3 \mathrm{~d}^{8}\left({ }^{3} \mathrm{~F}\right) 4 \mathrm{~d}$ & ${ }^{2} \mathrm{D}$ & -0.397851 & -0.397229 \\
\hline $3 d^{7} 4 s^{2}$ & ${ }^{4} \mathrm{P}$ & -0.718460 & -0.678817 & $3 d^{8}\left({ }^{3} F\right) 5 p$ & ${ }^{4} \mathrm{D}^{\mathrm{o}}$ & -0.387061 & -0.386626 \\
\hline $3 d^{8}\left({ }^{1} D\right) 4 p$ & ${ }^{2} \mathrm{D}^{\mathrm{o}}$ & -0.714643 & -0.694464 & $3 d^{8}\left({ }^{3} F\right) 5 p$ & ${ }^{2} \mathrm{G}^{\mathrm{O}}$ & -0.380284 & -0.380049 \\
\hline $3 d^{8}\left({ }^{1} D\right) 4 p$ & ${ }^{2} \mathrm{P}^{\mathrm{o}}$ & -0.712358 & -0.695635 & $3 d^{8}\left({ }^{3} F\right) 5 p$ & ${ }^{4} \mathrm{G}^{\mathrm{O}}$ & -0.376380 & -0.383804 \\
\hline $3 d^{7} 4 s^{2}$ & ${ }^{2} \mathrm{G}$ & -0.693374 & -0.654487 & $3 d^{8}\left({ }^{3} F\right) 5 p$ & ${ }^{4} \mathrm{~F}^{\circ}$ & -0.381231 & -0.383245 \\
\hline $3 d^{8}\left({ }^{3} P\right) 4 p$ & ${ }^{4} \mathrm{D}^{\mathrm{o}}$ & -0.694518 & -0.677702 & $3 d^{8}\left({ }^{3} F\right) 5 p$ & ${ }^{2} \mathrm{~F}^{\mathrm{o}}$ & -0.369686 & -0.374133 \\
\hline $3 d^{8}\left({ }^{3} P\right) 4 p$ & ${ }^{2} \mathrm{D}^{\mathrm{o}}$ & -0.682783 & -0.667664 & $3 d^{8}\left({ }^{3} F\right) 5 p$ & ${ }^{2} \mathrm{D}^{\circ}$ & -0.369733 & -0.372305 \\
\hline $3 d^{8}\left({ }^{3} P\right) 4 p$ & ${ }^{2} \mathrm{P}^{\mathrm{o}}$ & -0.671347 & -0.646032 & $3 \mathrm{~d}^{7}\left({ }^{4} \mathrm{P}\right) 4 \mathrm{~s} 4 \mathrm{p}\left({ }^{3} \mathrm{P}^{\mathrm{o}}\right)$ & ${ }^{6} \mathrm{D}^{\circ}$ & -0.373175 & -0.385229 \\
\hline $3 d^{7} 4 s^{2}$ & ${ }^{2} \mathrm{P}$ & -0.665580 & -0.606276 & $3 \mathrm{~d}^{8}\left({ }^{1} \mathrm{D}\right) 5 \mathrm{~s}$ & ${ }^{2} \mathrm{D}$ & -0.372491 & -0.368363 \\
\hline $3 d^{8}\left({ }^{3} P\right) 4 p$ & ${ }^{2} \mathrm{~S}^{\mathrm{o}}$ & -0.662030 & -0.646464 & $3 \mathrm{~d}^{7}\left({ }^{4} \mathrm{P}\right) 4 \mathrm{~s} 4 \mathrm{p}\left({ }^{3} \mathrm{P}^{\mathrm{o}}\right)$ & ${ }^{4} \mathrm{~S}^{\mathrm{O}}$ & -0.357170 & -0.352353 \\
\hline $3 d^{8}\left({ }^{3} P\right) 4 p$ & ${ }^{4} \mathrm{~S}^{\mathrm{o}}$ & -0.661870 & -0.634170 & $3 \mathrm{~d}^{8}\left({ }^{3} \mathrm{P}\right) 5 \mathrm{~s}$ & ${ }^{4} \mathrm{P}$ & -0.350365 & -0.346754 \\
\hline $3 d^{8}\left({ }^{1} G\right) 4 p$ & ${ }^{2} \mathrm{H}^{\mathrm{o}}$ & -0.653426 & -0.617867 & $3 \mathrm{~d}^{7}\left({ }^{4} \mathrm{P}\right) 4 \mathrm{~s} 4 \mathrm{p}\left({ }^{3} \mathrm{P}^{\mathrm{o}}\right)$ & ${ }^{6} \mathrm{P}^{\mathrm{o}}$ & -0.345315 & -0.354176 \\
\hline $3 d^{8}\left({ }^{1} G\right) 4 p$ & ${ }^{2} \mathrm{~F}^{\mathrm{o}}$ & -0.645270 & -0.620856 & $3 \mathrm{~d}^{7}\left({ }^{2} \mathrm{G}\right) 4 \mathrm{~s} 4 \mathrm{p}\left({ }^{3} \mathrm{P}^{\mathrm{o}}\right)$ & ${ }^{4} \mathrm{~F}^{\circ}$ & -0.337318 & -0.352432 \\
\hline $3 d^{7} 4 s^{2}$ & ${ }^{2} \mathrm{H}$ & -0.631814 & -0.591140 & $3 \mathrm{~d}^{8}\left({ }^{3} \mathrm{P}\right) 5 \mathrm{~s}$ & ${ }^{2} \mathrm{P}$ & -0.342100 & -0.337504 \\
\hline $3 d^{7} 4 s^{2}$ & ${ }^{2} \mathrm{D} 2$ & -0.628493 & -0.628492 & $3 \mathrm{~d}^{7}\left({ }^{4} \mathrm{~F}\right) 4 \mathrm{~s} 4 \mathrm{p}\left({ }^{1} \mathrm{P}^{\mathrm{o}}\right)$ & ${ }^{4} \mathrm{G}^{\mathrm{o}}$ & -0.305892 & -0.315432 \\
\hline $3 \mathrm{~d}^{8}\left({ }^{1} \mathrm{G}\right) 4 \mathrm{p}$ & ${ }^{2} \mathrm{G}^{\mathrm{o}}$ & -0.611035 & -0.574023 & $3 \mathrm{~d}^{8}\left({ }^{1} \mathrm{D}\right) 4 \mathrm{~d}$ & ${ }^{2} \mathrm{~F}$ & -0.311901 & -0.301290 \\
\hline $3 \mathrm{~d}^{7}\left({ }^{4} \mathrm{~F}\right) 4 \mathrm{~s} 4 \mathrm{p}\left({ }^{3} \mathrm{P}^{\mathrm{o}}\right)$ & ${ }^{6} \mathrm{~F}^{\mathrm{o}}$ & -0.540999 & -0.583215 & $3 d^{8}\left({ }^{1} D\right) 4 d$ & ${ }^{2} \mathrm{D}$ & -0.307279 & -0.297857 \\
\hline $3 \mathrm{~d}^{7}\left({ }^{4} \mathrm{~F}\right) 4 \mathrm{~s} 4 \mathrm{p}\left({ }^{3} \mathrm{P}^{\mathrm{o}}\right)$ & ${ }^{6} \mathrm{D}^{\mathrm{o}}$ & -0.531735 & -0.569387 & $3 \mathrm{~d}^{8}\left({ }^{1} \mathrm{D}\right) 4 \mathrm{~d}$ & ${ }^{2} \mathrm{G}$ & -0.307623 & -0.293094 \\
\hline $3 d^{8}\left({ }^{3} F\right) 5 s$ & ${ }^{4} \mathrm{~F}$ & -0.495178 & -0.491191 & $3 \mathrm{~d}^{8}\left({ }^{1} \mathrm{D}\right) 4 \mathrm{~d}$ & ${ }^{2} \mathrm{P}$ & -0.305995 & -0.296769 \\
\hline
\end{tabular}

wavefunctions, but may cause pseudoresonances, particularly if the two summations in Eq. (1) are inconsistent (Berrington et al. 1987). The present calculation includes all the configurations that result by adding an electron to the target configurations.

The whole calculation was divided into three groups of total (e + ion) symmetries $S L \pi$ according to their multiplicity, i.e. $(2 S+1)=2,4$, and 6 . For each multiplicity we consider total angular momenta $L=0-7$, for both parities.

\section{Results}

The results for the energy levels, oscillator strengths, and total and partial photoionization cross sections are described in the following sections.

\subsection{Energy levels of $\mathrm{Ni} I I$}

The calculations begin with the energies of 836 LS terms of Ni II corresponding to all possible bound states with principal quantum number $n \leq 10$. Table 2 shows some comparisons between the computed energies and experimental values from Sugar \& Corliss (1985). The energies 
Table 3. Comparison of calculated $f$-values in LS coupling for Ni II with experimental data from Fedchak \& Lawler (1999; FL), recommended values by Fuhr et al. (1988), and theoretical data from Kurucz \& Bell (1995; KB)

\begin{tabular}{lccccc}
\hline Configuration & Transition & Present & FL & Fuhr et al. & KB \\
\hline $3 \mathrm{~d}^{9}-3 \mathrm{~d}^{8}\left({ }^{3} \mathrm{~F}\right) 4 \mathrm{p}$ & ${ }^{2} \mathrm{D}-{ }^{2} \mathrm{~F}^{\circ}$ & 0.0465 & 0.0430 & - & 0.182 \\
$3 \mathrm{~d}^{9}-3 \mathrm{~d}^{8}\left({ }^{3} \mathrm{~F}\right) 4 \mathrm{p}$ & ${ }^{2} \mathrm{D}-{ }^{2} \mathrm{D}^{\circ}$ & 0.0970 & - & - & 0.294 \\
$3 \mathrm{~d}^{9}-3 \mathrm{~d}^{8}\left({ }^{1} \mathrm{D}\right) 4 \mathrm{p}$ & ${ }^{2} \mathrm{D}-{ }^{2} \mathrm{~F}^{\circ}$ & 0.0218 & - & - & 0.0483 \\
$3 \mathrm{~d}^{9}-3 \mathrm{~d}^{8}\left({ }^{1} \mathrm{D}\right) 4 \mathrm{p}$ & ${ }^{2} \mathrm{D}-{ }^{2} \mathrm{D}^{\circ}$ & 0.0310 & - & - & 0.124 \\
$3 \mathrm{~d}^{9}-3 \mathrm{~d}^{8}\left({ }^{3} \mathrm{P}\right) 4 \mathrm{p}$ & ${ }^{2} \mathrm{D}-2{ }^{\circ} \mathrm{D}^{\circ}$ & 0.0244 & - & - & 0.0898 \\
$3 \mathrm{~d}^{9}-3 \mathrm{~d}^{8}\left({ }^{3} \mathrm{P}\right) 4 \mathrm{p}$ & ${ }^{2} \mathrm{D}-{ }^{2} \mathrm{P}^{\circ}$ & 0.0917 & - & - & 0.261 \\
$3 \mathrm{~d}^{9}-3 \mathrm{~d}^{8}\left({ }^{3} \mathrm{~F}\right) 4 \mathrm{p}$ & ${ }^{2} \mathrm{D}-{ }^{2} \mathrm{~F}^{\circ}$ & 0.0801 & - & - & 0.312 \\
$3 \mathrm{~d}^{8}\left({ }^{3} \mathrm{~F}\right) 4 \mathrm{~s}-3 \mathrm{~d}^{8}\left({ }^{3} \mathrm{~F}\right) 4 \mathrm{p}$ & ${ }^{4} \mathrm{~F}-{ }^{4} \mathrm{~F}^{\circ}$ & 0.246 & 0.218 & 0.222 & 0.487 \\
& ${ }^{4} \mathrm{~F}-{ }^{4} \mathrm{G}^{\circ}$ & 0.344 & 0.307 & - & 0.221 \\
& ${ }^{2} \mathrm{~F}-{ }^{2} \mathrm{G}^{\circ}$ & 0.329 & 0.240 & 0.248 & 0.248 \\
& ${ }^{2} \mathrm{~F}-{ }^{2} \mathrm{~F}^{\circ}$ & 0.247 & 0.234 & 0.312 & 0.226 \\
& ${ }^{2} \mathrm{~F}-{ }^{2} \mathrm{D}^{\circ}$ & 0.183 & - & 0.166 & 0.166 \\
\hline
\end{tabular}

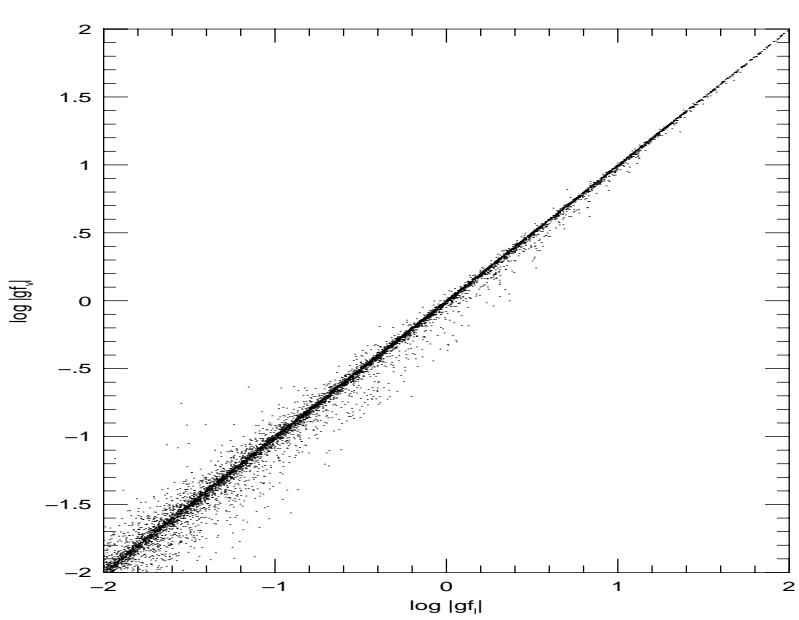

Fig. 1. $\log g f_{V}$ plotted against $\log g f_{L}$ for transitions between calculated LS terms

obtained in the present work agree with the experimental values typically within $2 \%$ throughout the entire data set with the only exception of the equivalent electron states of the form $3 \mathrm{~d}^{7} 4 \mathrm{~s}^{2}$. For these states, the calculated energies differ from experiment by about $6 \%$. It is also found that near the Ni II ionization limit, where the density of states is large, there are numerous mismatches between the identification of states in the Sugar \& Corliss compilation and the present results. The present level identifications are based on both percentage channel contributions and quantum defects and they do seem to be secure. Thus, more experimental work on the detection and proper identification of highly excited state is needed.

\subsection{Oscillator strengths}

Dipole oscillator strengths ( $f$-values) for 23738 transitions among the calculated states of Ni II were obtained in LS
Table 4. Comparison of calculated radiative lifetimes (in $10^{-9} \mathrm{~s}$ ) of Ni II states with experimental data from Fedchak \& Lawler (1999; FL) and theoretical values from Kurucz \& Bell (1995)

\begin{tabular}{llccc}
\hline Configuration & Multiplet & Present & FL & KB \\
\hline $3 \mathrm{~d}^{8}\left({ }^{3} \mathrm{~F}\right) 4 \mathrm{p}$ & $\mathrm{z}{ }^{4} \mathrm{D}^{\circ}$ & 3.235 & 3.24 & 3.068 \\
& $\mathrm{z}{ }^{4} \mathrm{G}^{\circ}$ & 2.886 & 2.96 & 3.057 \\
& $\mathrm{z}{ }^{4} \mathrm{~F}^{\circ}$ & 2.971 & 2.96 & 2.882 \\
& $\mathrm{z}{ }^{2} \mathrm{G}^{\mathrm{o}}$ & 3.392 & 3.11 & 3.105 \\
& $\mathrm{z}{ }^{2} \mathrm{~F}^{\mathrm{o}}$ & 2.810 & 2.51 & 2.313 \\
& $\mathrm{z}{ }^{2} \mathrm{D}^{\mathrm{o}}$ & 1.951 & 2.00 & 1.564 \\
\hline
\end{tabular}

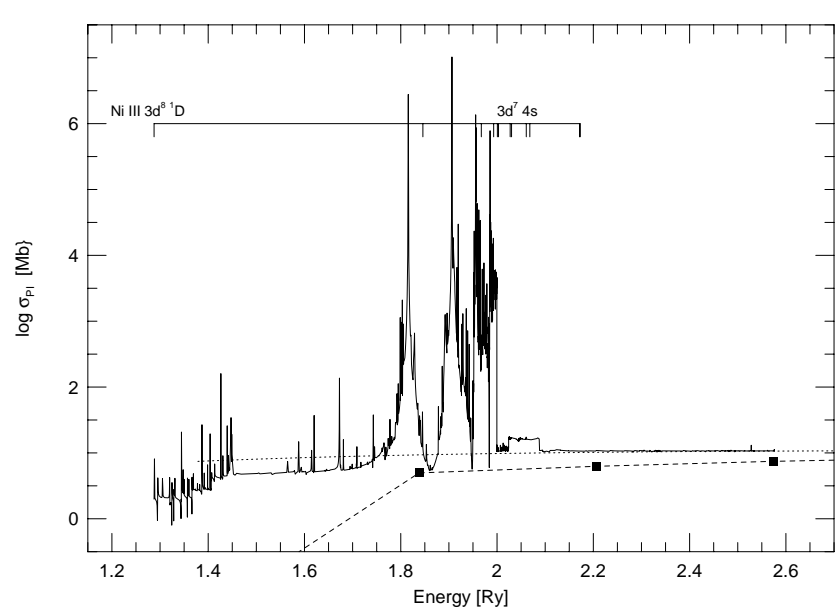

Fig. 2. Photoionization cross section $(\sigma(\mathrm{Mb}))$ of the ground state $3 \mathrm{~d}^{9}\left({ }^{2} \mathrm{D}\right)$ of $\mathrm{Ni}$ II as a function of photon energy (Rydbergs). The dotted curve shows the results of Verner et al. (1993) and the filled squares, those of Reilman \& Manson (1979) 

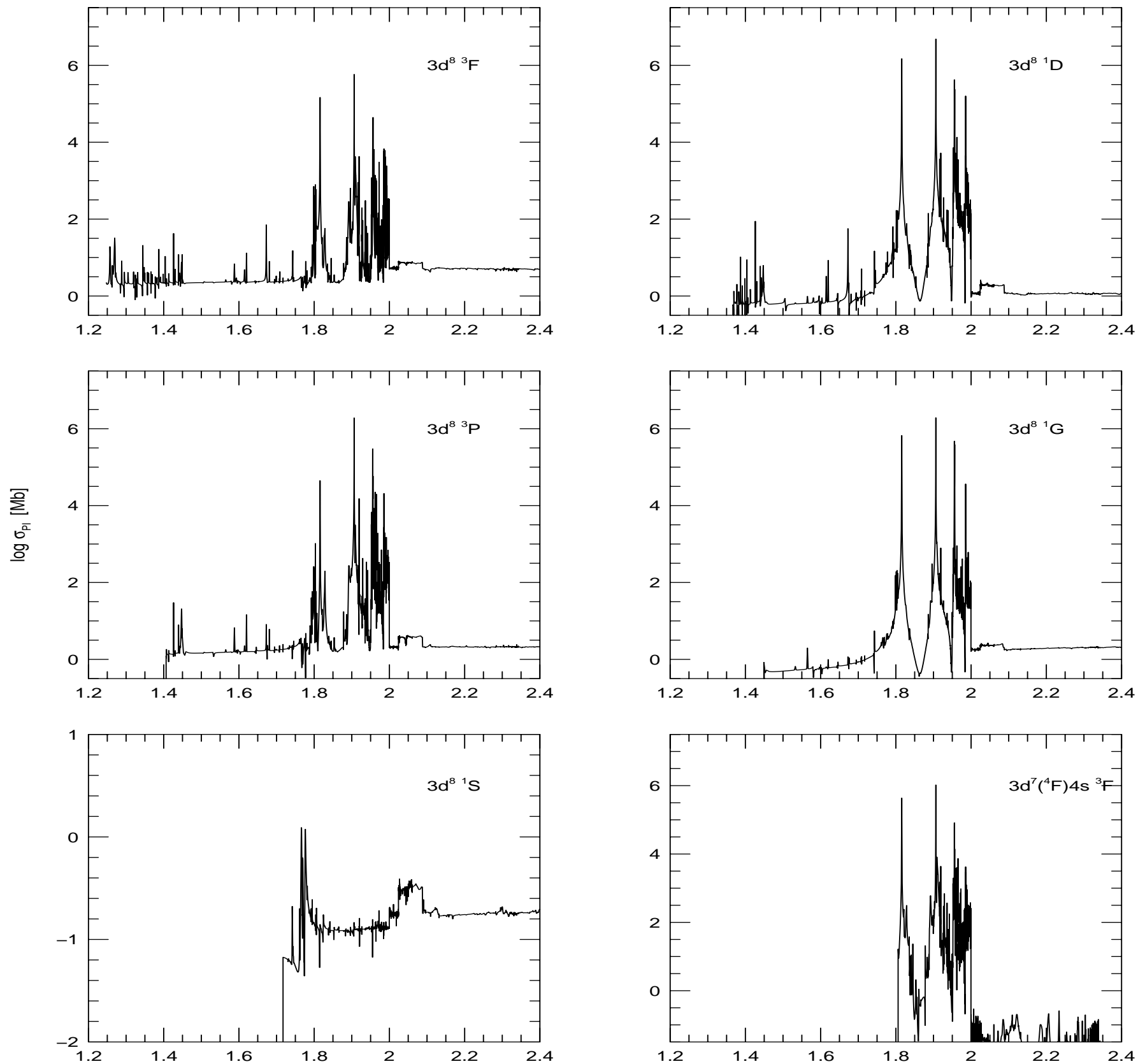

Energy [Ry]

Fig. 3. Photoionization cross section $(\sigma(\mathrm{Mb}))$ of excited states of Ni II as a function of photon energy (Rydbergs)

coupling. This set includes transitions for which the lower state lies below the first ionization threshold and the upper state lies above. These transitions can be important in opacity calculations because they contribute to the total photo-absorption, but do not appear as resonances in the photoionization cross sections (strictly speaking, the upper bound state does autoionize if departure from LS coupling is considered and fine structure continua are explicitly allowed).
Comparison of length and velocity oscillator strengths provides a systematic consistency check on the accuracy of the wavefunctions and, therefore, on the reliability of the $f$-values. In Fig. 1 we plot $\log \left(g f_{V}\right)$ vs. $\log \left(g f_{L}\right)$. We have included all the symmetries since each exhibits roughly the same dispersion. The dispersion between length and velocity values is $\sim 9 \%$ for $g f$-values greater than unity and $\sim 12 \%$ for $g f$-values greater than 0.1 . 

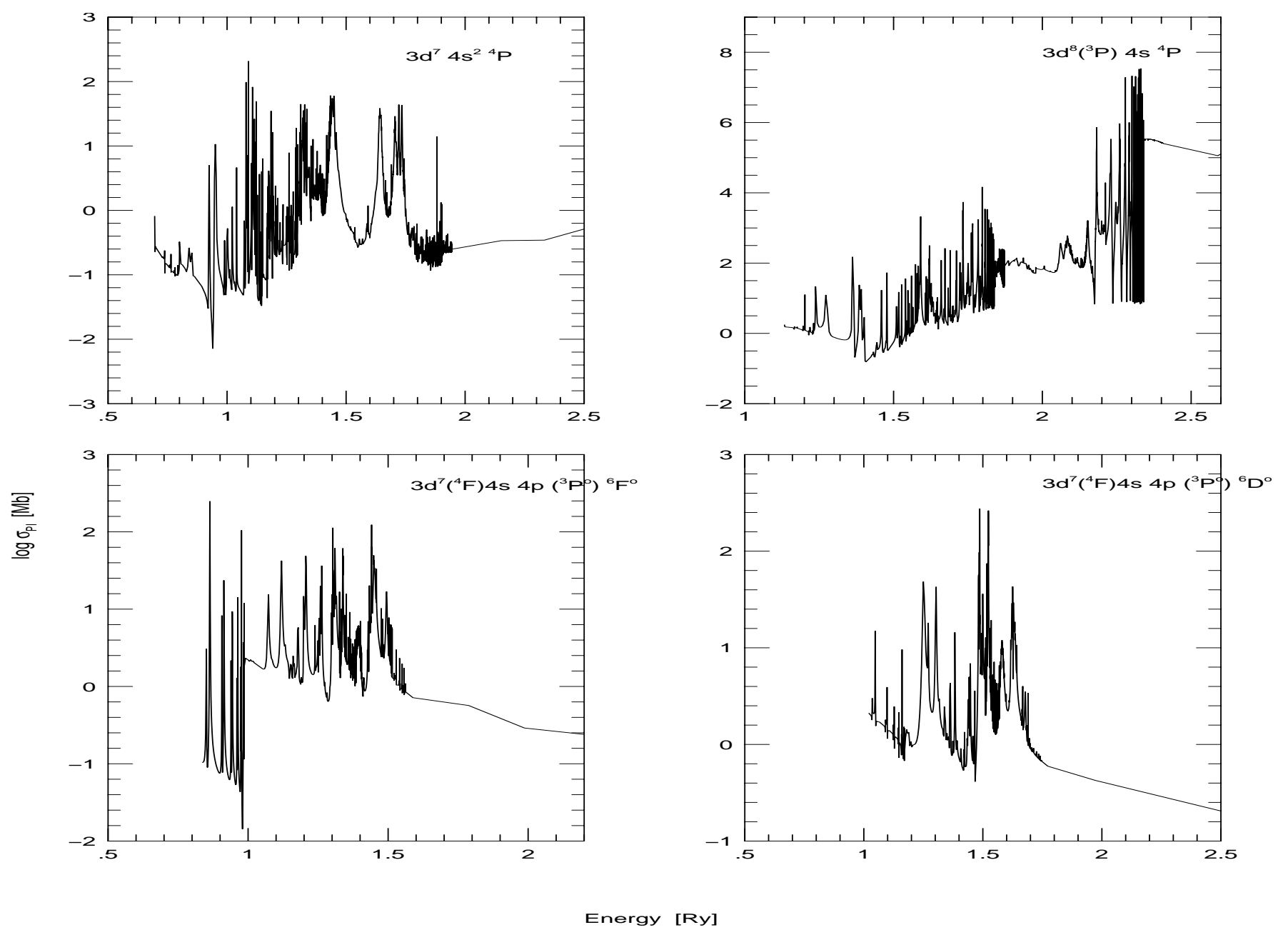

Fig. 4. Partial photoionization cross sections of the Ni II ground state going into the lowest six states of Ni III

The first experimental determination of Ni II $f$-values in the VUV was recently reported by Fedchak \& Lawler (1999). While this work awaits publication all determinations of Ni II abundance from absorption lines in the diffuse ISM (e.g. Morton 1991; Zsargó \& Federman 1998) have been based on theoretical data by Kurucz \& Peytremann (1975) and Kurucz \& Bell (1995).

Table 3 presents a comparison of the present $f$-values with the experimental data from Fedchak \& Lawler (1999), the recommended values by Fuhr et al. (1988), and those from semiempirical computations of Kurucz \& Bell (1995). Notice the recommended data by Fuhr et al. is mostly based on the values of Kurucz \& Peytremann adjusted such as the radiative lifetime of the levels agreed with a few experimental measurements. Table 4 compares radiative lifetimes obtained from the present data with those measured by Fedchak \& Lawler and calculated using the Kurucz \& Bell data.

The tables show good agreement between the present data and the experimental values of Fedchak \& Lawler but, there are significant differences with respect to the $f$-values of Kurucz \& Bell and Fuhr et al. Particular discrepancies exist for transitions that involve the $3 \mathrm{~d}^{9}{ }^{2} \mathrm{D}$ ground state of Ni II. For these the Kurucz \& Bell $f$-values seem to be overestimated by factors of two to four. These transitions are also the ones commonly observed from spectra of the ISM; furthermore, the gas phase abundance of nickel in the ISM maybe higher than previously estimated.

The good agreement between length and velocity $f$-values and the agreement for both absolute $f$-values and level lifetimes with experimental determinations suggests that the overall uncertainty for such transitions should be near $10 \%$. However, weaker transitions are likely to have greater uncertainties. For weak transitions relativistic effects, not included here, may be important. Similarly, algebraic splitting of the present $f$-values in LS coupling into fine structure $f$-values would lead to large errors for relativistic effects are quite important at the fine structure level in Ni II. 


\subsection{Photoionization cross sections}

Photoionization cross sections were calculated for all bound states. These cross sections include detailed autoionization resonances. Figure 2 shows the photoionization cross section of the $\left(3 \mathrm{~d}^{9}{ }^{2} \mathrm{D}\right)$ ground state of Ni II. In the same figure we have plotted the results of Verner et al. (1993) and Reilman \& Manson (1979), both using central field type approximations. One interesting feature in the present cross section is the packs of large resonances between 1.75 and 2 Ryd that rise several orders of magnitude above the background. These resonances result from the coupling of the ground state $\left(3 \mathrm{~d}^{8}{ }^{1} \mathrm{D}\right)$ with the excited state of the form $\left(3 d^{7} 4 s\right)$ of the Ni III target. The failure of other authors to obtain resonance structures in the cross sections is due to the absence of the relevant electron correlations in those calculations. This is always the case for the central field approximations.

Figure 3 shows the photoionization cross sections for a few excited states of Ni II.

In addition to the total photoionization cross sections we obtained also partial cross sections for photoionization going into each of the states of the target ion. These cross sections are needed for the computation of recombination rates (Nahar \& Pradhan 1995) and in constructing nonLTE spectral models where it may be important to determine accurately the populations of excited levels of the residual ion following photoionization. Figure 4 presents these partial cross sections for the Ni II ground state going into into the lowest six levels of Ni III.

\section{Conclusion}

Extensive radiative calculations for $\mathrm{Ni}$ II are presented which are expected to be applicable to a variety of astrophysical problems such as the calculation of improved opacities, non-LTE models, and spectroscopic interpretation of Ni II spectra. The present calculations include a large number of target terms and therefore exhibit resonance structures absent from previous calculations. Also presented are oscillator strengths for 23738 dipole allowed transitions among the terms in Ni II.

The current $f$-values and the level radiative lifetimes derived from them agree rather well with available experimental measurements. In contrast, there are significant differences with respect to previous calculations by Kurucz and coworkers using semiempirical methods. These differences reach factors of two to four for transitions commonly used in Ni II abundance determinations in the ISM. Thus, previous estimates of nickel depletion in the ISM may be overestimated. The present data is also expected to have important effects on the Ni II opacities.

All radiative data reported here will be included in TOPbase at CDS (Cunto et al. 1993). These data can also be accessed electronically via Internet by request to the authors.

Acknowledgements. I like to thank Dr. J.A. Fedchal and Dr. J.E. Lawler for providing me with their experimental measurements prior to publication. I also like to thank Prof. A.K. Pradhan and Prof. David G. Hummer for useful comments and reading of the manuscript. The computations were carried out on the CRAY J94 at the NASA Center for Computational Sciences and the CRAY T3E at the High Performance Computing and Communications Program at NASA/GSFC.

\section{References}

Berrington K.A., Burke P.G., Butler K., et al., 1987, J. Phys. B: At. Mol. Phys. 20, 6379

Cunto W., Mendoza C., Ochsenbein F., Zeippen C.J., 1993, A\&A 275, L5-L8

Eissner W., Jones M., Nussbaumer H., 1974, Comput. Phys. Commun. 8, 270

Eissner W., 1991, J. Phys. IV (Paris) C1, 3

Fuhr J.R., Martin G.A., Wiese W.L., 1988, J. Phys. Chem. Ref. Data 17, Suppl. 4

Fedchal J.A., Lawler J.E., 1999, ApJ (submitted)

Hass S., Dreizler S., Heber U., Jeffery S., Werner K., 1996, A\&A 311, 669

Henry R.B.C., Fesen R.A., 1988, ApJ 329, 693

Hummer D.G., Berrington K.A., Eissner W., et al., 1993, A\&A 279, 298 (Paper I)

Kurucz R.L., Bell B., 1995, KURUCZ CD-ROM 23, 1995, update of the original calculations of Kurucz R.L. \& Peytremann E. (eds.) 1975

Kurucz R.L., Peytremann E., 1975, Smithsonian Astrophys. Obs. Spec. Rep. 362, 197

Morton D.C., 1991, ApJS 77, 119

Nahar S.N., 1995, A\&AS 293, 967 (Paper VII)

Oliva E., Drapatz S., Lutz D., Sturm E., Moorwood A.F.M., 1988, Ap\&SS 255, 2110

Reilman R.F., Manson S.T., 1979, ApJS 40, 815

Seaton M.J., Yu Yan, Mihalas D., Pradhan A.K., 1994, MNRAS 266, 805

Sugar J., Corliss C., 1985, J. Phys. Chem. Ref. Data 14 Suppl., No. 2

Verner D.A., Yakovlev D.G., Band I.M., Trzhaskovskaya M.B., 1993, At. Data. Nucl. Data Tab. 55, 233

Yu Yan, Seaton M.J., 1987, J. Phys. B: At. Mol. Phys. 20, 6409

Zsargó J., Federman S.R., 1998, 498, 256 